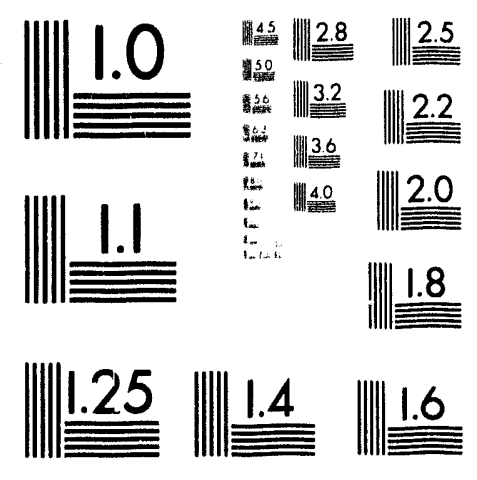



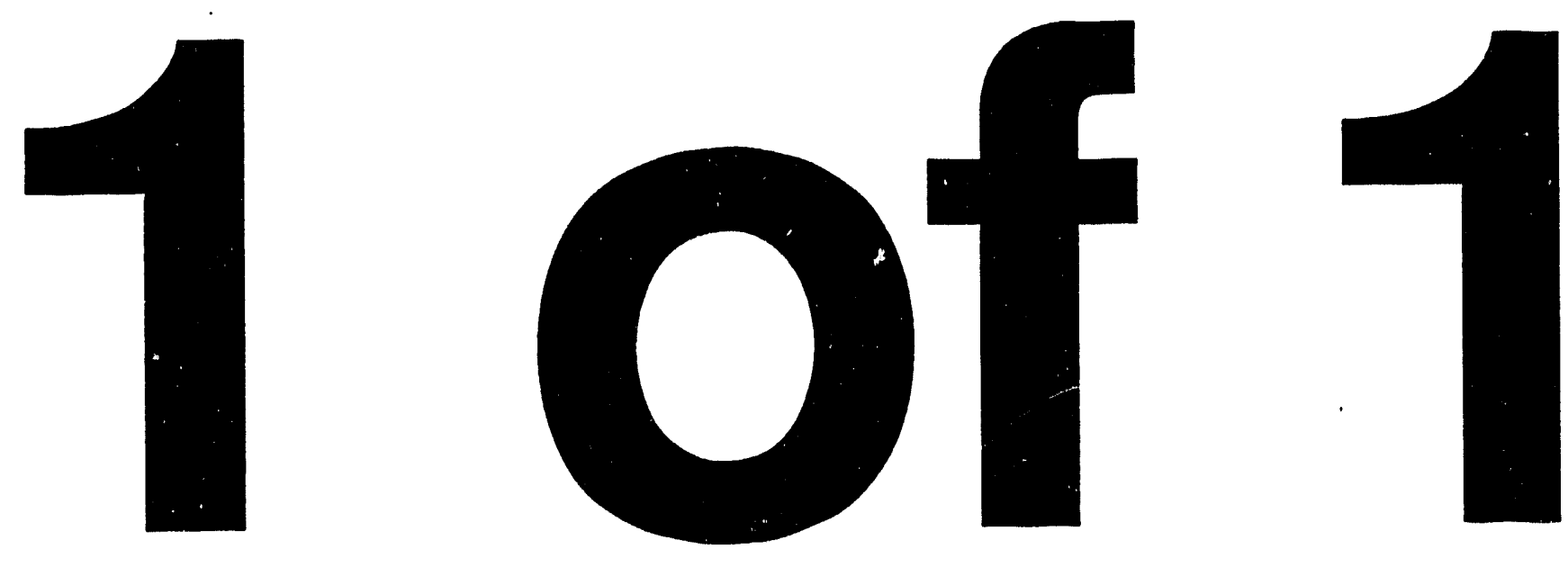
Manuscript for 3rd Workshop on Advanced Accelerator Concepts

Port Jefferson, New York, 14-20 June 1992.

\title{
PHOTONIC BAND GAP STRUCTURES: A NEW APPROACH TO ACCELERATOR CAVITIES
}

\author{
N. Kroll,* D. R. Smith, and S. Schuliz. \\ Department of Phy'sics, University of California, San Dicgo \\ 9500 Gilman Drive, La Jolla, California 92093-(0319 \\ ABSTRACT \\ ASO3 - 89ER 40527
}

We introduce a new accelerator cavity design based on Photonic Band Gap (PBG) structures. The PBG cavity consists of a two-dimensional periodic array of high dielectric, low loss cylinders wilh a single removal defect, bounded on top and bottom by conducting sheets. We present the results of both numerical simulations and experimental measurements on the PBG cavity.

\section{INTRODUCTION}

We consider here a new class of resonant cavitics which we are investiganing in the context of what has come to be termed Photonic Band Gap (PBG) structures. ${ }^{1,2}$ The concepts underlying the PBG condition, and the experimental and numerical confirmation of typical resonant modes, will be prescnted later in this paper. The configurations we have investigated are comprised of a periodic array of short dielectric cylinders, which are bounded on top and bottom by a metal sheet, and from which one cylinder has been removed at the central site. We find that the electromagnetic modes associated with these configurations are analogous to resonant cavity modes, with the E field polarized perpendicular to the metal sheets. Thus, if an aperture is placed in each metal sheet at the sile of the missing cylinder, we can envision an electron beam entering through one plate, bcing accelernted by the parallel of $E$ field, and then emerging from the other plate. A suitable three-section coupled set of such structures could, for example, form the familiar $2 \pi$ basic accelerator unit with $2 \pi / 3$ phase advance per section (Fig. 1).

The reasons why we believe this new class of structures may be particularly suited for acceleralors are:

(i) They have a set of resonant frequencies which are entirely different from those associnted with the usual cavily structures, and therefore may be much better suited to reducing the lingher order mode problem. Indeed, it may be possible to arrange that there will be only one trapped mode.

(ii) The spatial distribution of the $E$ field for the resonant mode falls off exponentially from the center of the active region, thus minimizing constraints on the boundary material.

(iii) The structures may be readily fabricated utilizing sapphire cylinders and superconducting niobium plates which will result in $Q$ values $>10^{6}$. We note that the only superconducting material renuired is in the form of a flat sheet, with no bends, joints, or welds, a circumstance which may mitigate limitations of acceleration gradient associated with the superconducting surface.

*Also Stanford Linear Accelerator Center, Stanford University, Stanford CA 94309 process disclosed, or represents that its use would not infringe privately owned rights. Reference herein to any specific commercial product, process, or service by trade name, trademark, manufacturer, or otherwise does not necessarily constitute or imply its endorsement, recommendation, or favoring by the United States Government or any agency thereof. The views and opinions of authors expressed herein do not necessarily state or reflect those of the 


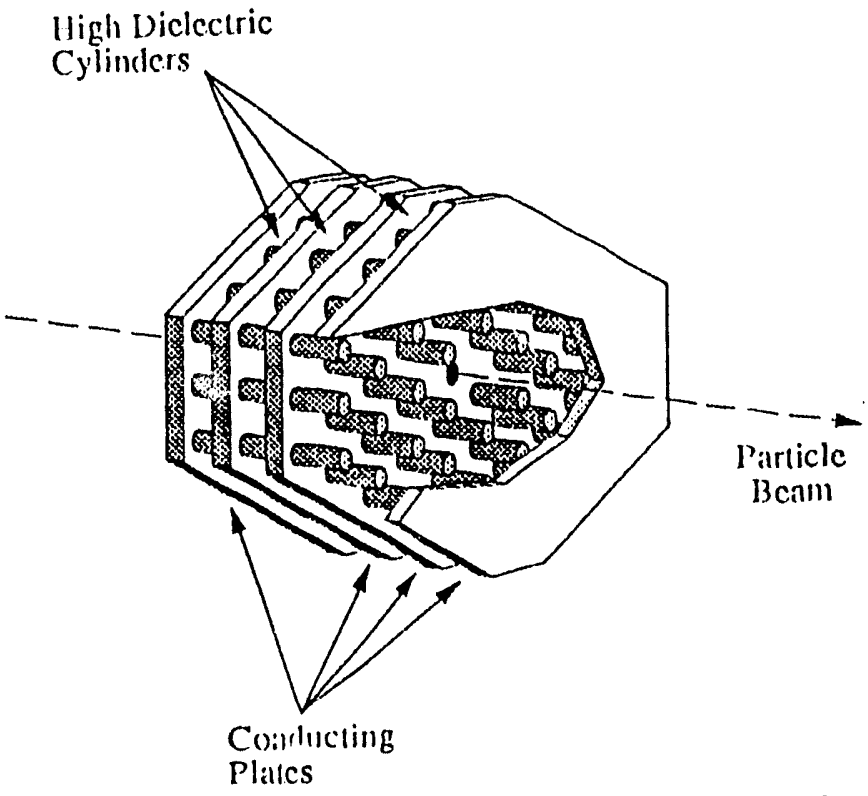

Fig. 1. A schematic view of the proposed $2 \pi$ accelerator unit. In this example the unit consists of three triangular photonic lattices, separated by superconducting sheets. Each of the lattices has a cylinder removed to allow the formation of a defect mode with an electric field maximum in the center. Holes drilled through the conducting plates would allow a particle beam to be accelerated through the unit.

We envisage a number of potential problems with the proposed structures. In particular, there is the concern that dielectric material (such as pure sarphire) located inside the vacumm, and at a distance of $\sim 1 \mathrm{~cm}$ from the electron beam will exhibit charging, multipaction, and/or dielectric breakdown. Furthermore, we have not studied problems which may be associated with junctions between the dielectric and the superconducting sheets. It will require further numerical simulation and the appropriate experimental testing to establish the optimum configurations and validity of this approach.

In Section I we present a physical explanation of the PI3G concept. In Section II we discuss our experimental equipment and techniques. In Section Ill we discuss the methodology that has been formulated for the numerical determination of the electromagnetic "band structure", and also calculation of the defect states. In Section IV we present some of the experimental results which illustrate the key properties of selected PBG-defect structures. Some concluding discussion and remarks are given in Section V.

\section{Introduction to the PBG Concept}

It is simplest to introduce the PBG concept in the context of wave propagation in physical systems which have a periodic structure along some specified spatial direction. Well known examples are the wave function of a particle moving in a periodic polcntial, mode propagntion in periodic linear acceleralor structures, and a 
waveguide of uniform cross section filled with dielectric with permiltivity varying periodically along the waveguide axis. For concreteness we fix our attention on the last of these and recall that such sy'stems can be discussed in terms of modes which are fully charncterized by the transverse variation of their electromagnetic fields along the waveguide cross section. Fixing our allention on a particular mode we note that such a struclure will cxhibit an allemating scrics of stop bands and pass bands as the frequency is varied. ${ }^{3}$ In the language of the PBG community the stop bands are referred to as photonic band gaps; photonic because we are dealing with Maxwell's equations (not entirely logical because we are dealing with them classically) and bands gaps in analogy with the language used 10 describe electronic stales in the periodic potentials of crystalline solids. A wave propagating in a dielectric free section of the waveguide which encounters a long section of the periodic structure will be tolally reflected if its frequency lies within one of the band gaps. Thus, if one intcrupts the periodicity by removing a section of the periodically varying dielectric, there is the possibility of a trapped mode in which a wave whose frequency lies in the band gap is reflecicd back and forth belween the (wo dielectric filled sections; if the multiply reflected waves are in phase with one another then the possibility is realized. The resultant trapped mode is reforred 10 as a defect state, associated with the fact hat the gap in the periodicity is referred to as a defect, both in analogy with the terminology applied to electronic stales in solids.

Before proceeding to a discussion of the case of periodicity in more than one dimension, we brictly mention an extension of the above discussion to the case of structures which are symmetric under rotation about an axis with dielectric constant which varies as a function of the radial distance $r$ from the axis. ${ }^{4}$ For example, one might consider a set of contiguous concentric tubes of alternating dielectric constant. It is easy to show that one can choose the radii of the cylinders so as to have bands of frequencies that are non-propagating for rotationally symmetric radial waves. By omitting an appropriate amount of the central section one can again have radial waves with frequency in the gap which experience multiple reflections from the surrounding structure. Again, if the dimensions are such that the multiple reflections are in phase, one has a trapped mode. One can rendily envisage a mode of this sort which would be quite suitable for particle acceleration. We refer 10 such configurations as "radial band gap" structures with radial band gap modes.

We tum now to consideration of structures with two-dimensional periodicity of the sort discussed in the introduction. We first focus our attention on the case of an infinite periodic lattice. The solutions of Maxwell's cquations can be chosen such that each component has the form $\exp (i \bar{k} \cdot \bar{R}) F(\bar{R}) G(z)$ where the functions $F$ have the periodicity of the lattice. Here $R$ is a two-dimensional position vector in the plane of periodicity and $z$ the coordinate perpendicular to it. The two-dimensional vector $k$ is referred to as the wave vector and may be thought of as residing in the reciprocal lattice space. For each value of $k$ there are a discrete set of solutions with a discrete set of frequencies $\left\{\omega_{n}(k)\right\}$. The band index $n$ refers to the particular discrete solution. The $\left\{\omega_{n}\right\}$, which may be thought of as two-dimensional surfaces in $(k, \omega)$ space, have the double periodicity of the reciprocal lattice. Each ()$_{11}$ and the associated solutions represents an allowed frequency band of propagating waves. The frequency span associated with each band surface may or may not overlap that associated with other surfaces. Hence, in contrast with the one-dimensional case, there may or may not be "band gaps", that is, frequency ranges for which no propagating solutions exist. As the name suggests, a PBG system is an array which does contain band gaps.

A "defect", formed, saly by removing one of the cylinders, presents the same possibility that we noted in the one-dimensional situation, namely a superposition of plane waves with frequency in the gap which are reflected into one another when they 
try to penetrate the surrounding lattice. For particular geometries and frequency these multiple reflections may be in-phase so as to allow them to combine to form a trapped mode, analogous to the localized electronic defect states familiar in semiconductor physics.

Experimental and theoretical studies of band structure and trapped localized defect modes in two-dimensional structures will be presented in the next sections.

\section{EXPERIMENTAL APPARATUS}

In order to experimentally study the band gap stuclure and associated defect modes, we utilize a microwave scattering chamber, ${ }^{4}$ which enables us 10 make mappings of the standing wave defect modes, and also measure the microwave transmission through various lattices of dielectric cylinders. The irterior of the scattering chamber is $1 \mathrm{~cm}$ high, $46 \mathrm{~cm}$ wide, and $51 \mathrm{~cm}$ long. The bottom and side walls of the chamber are machined out of a solid aluminum plate. On both ends of the chamber are standard 8-12 GHz waveguide filtings which can be used to delect or inject microwaves in the chamber via a tapered region inlegrally machined into the main plate. An aluminum cover plate, frec to move laterally, completes the chamber. The scatterers inside the chamber are typically cylinders with height of $1 \mathrm{~cm}$ and a variety of radii and dielectric constants. Accurate lattices can be constructed by placing the cylinders into a precision drilled styrofoam (dielectric constant of 1.(03) template. Finally, a thick layer of low density absorber is placed between the interior chamber walls and the styrofoam template, which serves to minimize reflection.

In conjunction with an HP network analyzer we are able to sweep the microwave frequency and make measurements of the power transmitted through the scaltering region. We are also able to map the spatial structure of standing wave modes (e.g., defect modes) by weakly coupling to a tuned probe through any of a lattice of small holes in the cover plate. Using standard homodyne techniques, we can measure both the phase and amplitudes of the fields sampled by the probe. By mapping the Mie resonances associated with scattering from a single cylinder, we have found that the probe does not significantly perturb the system and that the chamber is adequately terminated.

\section{II. THEORETICAL CALCULATIONS}

\section{^. Bandstructure}

A great deal of work has recently been done to compule the band structure for a lattice of infinitely long dielectric cylinders. ${ }^{5,6,7}$ These calculations have mainly been concerned with the specific case of waves propagating perpendicular to the cylinder axes; that is to say, modes for which the fields are independent of $\mathrm{z}$, where $\mathrm{z}$ is the coordinate axis parallel to the cylinder axes. For this case modes with electric field polarization parallel and perpendicular to the cylindrical axis propagate independently. Equivalently, the modes can be characterized as transverse magnetic (TM) and transverse electric (TE) with respect to the $z$-axis. In order to discuss the full set of modes for the accelerator cavity which we have in mind, it is necessary to 
cxtend these calculations to include arbitrary propagation direction so that fields are assumed to have an $\exp (\mathrm{iqz}$ ) variation, $\mathrm{q}$ being the wavevector directed along the cylinder axes. This causes the TE and TM modes to hybridize and to lead to a coupled pair of second order 2-D partial differential equations instead of the uncoupled pair which occur for the $\mathrm{g}=0$ case.

Maxwell's equations for the lattice of dielectric cylinders, taken to be infinite in the transverse directions, are

$$
\begin{aligned}
& \vec{\nabla} \times \vec{E}=-i \frac{\omega}{c} \mu \vec{H} \\
& \vec{\nabla} \times \vec{H} \doteq i \frac{\omega}{c} \varepsilon \vec{E}
\end{aligned}
$$

where $\varepsilon(x, y)$ is the periodic dielectric function representing the cylinders, and $\mu=1$ everywhere. We have assumed a time dependence of $\mathrm{e}^{i \omega l}$. If we further assume an explicit dependence on the longitudinal (z) component such that

$$
\begin{aligned}
& \vec{E}=\left(\vec{E}_{1}+\hat{z} E\right) e^{-i q x} \\
& \vec{H}=\left(\vec{H}_{1}+\hat{z} H\right) e^{-i q z}
\end{aligned}
$$

then Eqs. (1) and (2) yield

$$
\begin{aligned}
& -i q \hat{z} \times \vec{E}_{1}-\hat{z} \times \vec{\nabla} E=-i \frac{\omega}{c} \mu \vec{H}_{1} \\
& \vec{\nabla} \times \vec{E}_{1}=-i \frac{\omega}{c} \mu H \hat{z} \\
& -i q \hat{z} \times \vec{H}_{1}-\hat{z} \times \vec{\nabla} H=i \frac{\omega}{c} E \vec{E}_{1} \\
& \vec{\nabla} \times \vec{H}_{1}=i \frac{\omega}{c} \varepsilon E \hat{z}
\end{aligned}
$$

Eqs. (5) through (8) can be combined to yield

$$
\vec{\nabla} \cdot \varepsilon \alpha \vec{\nabla} E+\varepsilon \kappa^{2}=\beta \hat{z} \cdot \vec{\nabla} \times \alpha \vec{\nabla} H
$$

where

$$
\vec{\nabla} \cdot \alpha \vec{\nabla} H+\kappa^{2}=-\beta \hat{z} \cdot \vec{\nabla} \times \alpha \vec{\nabla} E
$$




$$
\begin{aligned}
& \kappa^{2}=\frac{\omega^{2}}{c^{2}}-q^{2} \\
& \beta=\frac{q c}{\omega} \\
& \alpha=\frac{1-\beta^{2}}{\varepsilon-\beta^{2}}
\end{aligned}
$$

The transverse field components have been eliminated in Egs. (9) and (10), and we are thus left with coupled linear equations for the $z$ field components $E(x, y)$ and $H(x, y)$ with parameter $\beta$.

Since $\varepsilon(x, y)$ is invariant under translation by any distance composed of integral multiples of a lattice constant, we ma expand it in an infinite sum over reciprocal latlice vectors as

$$
\varepsilon(\overrightarrow{\mathbf{x}})=\sum_{\dot{0}} \varepsilon_{\overrightarrow{\mathrm{o}}} \mathrm{e}^{\mathrm{i} \overrightarrow{\mathrm{u}} \cdot \overrightarrow{\mathrm{x}}}
$$

where

$$
\overrightarrow{\mathrm{G}} \cdot \vec{R}=2 \pi n \quad n=0, \pm 1, \pm 2, \ldots
$$

and

$$
\tilde{\varepsilon}_{\overline{\mathrm{o}}}=\delta_{\overline{\mathrm{o}, 0}}+(\varepsilon,-1) \mathrm{f} \frac{2 \mathrm{~J}_{1}(|\overrightarrow{\mathrm{G}}| \mathrm{a})}{|\overrightarrow{\mathrm{G}}| \mathrm{a}}
$$

$f$ is the filling factor, $\varepsilon_{a}$ is the cylinder dielectric constant, $a$ is the cylinder radius, and $R$ is a translation vector. $\alpha(x, y)$ can be expanded similarly. (For future reference we note that $1 / \varepsilon(x, y)$, which we will need later, may also be expanded in this way.) The translational invariance also restricts the field solutions of Eqs. (9) and (10) to satisfy

$$
\begin{aligned}
& E(\vec{x}+\vec{R})=e^{i \vec{k} \cdot \vec{k}} E(\vec{x}) \\
& H(\vec{x}+\vec{R})=e^{i \vec{k} \cdot \vec{k}} H(\vec{x})
\end{aligned}
$$

We may thus expand the field solutions in terms of reciprocal lattice vectors as 


$$
\begin{aligned}
& E(\vec{x})=\sum_{\vec{j}} E_{\vec{i}} e^{i(\vec{k}+\vec{i}) \cdot \vec{x}} \\
& H(\vec{x})=\sum_{\vec{j}} H_{\tilde{G}} e^{i(\vec{k}+\vec{G}) \cdot \vec{x}}
\end{aligned}
$$

$\mathrm{k}$ is known as the wave vector, or crystal momentum in solid state physics; for an infinite system with translational symmetry, $\mathrm{k}$ indexes the allowed eigenmodes, which are known as Bloch states, or rumning wave states. (We are thinking of the states as Bloch states in 2-D modulated by $\exp (\mathrm{iqz})$.) Substituting all the above expansions into Eqs. (9) and (10), we arrive at the following coupled equations:

$$
\begin{aligned}
& \sum_{\overrightarrow{\mathrm{G}}^{\prime}} \tilde{\alpha}_{\overrightarrow{\mathrm{o}}-\overline{\mathrm{G}}^{\prime}}\left[(\overrightarrow{\mathrm{k}}+\overrightarrow{\mathrm{G}}) \cdot\left(\vec{k}+\overrightarrow{\mathrm{G}}^{\prime}\right) \tilde{\mathrm{H}}_{\overline{\mathrm{G}}^{\prime}}+\beta \hat{z} \cdot(\vec{k}+\overrightarrow{\mathrm{G}}) \times\left(\vec{k}+\overrightarrow{\mathrm{G}}^{\prime}\right)\right] \tilde{\mathrm{E}}_{\tilde{\mathrm{c}}^{\prime}}=\kappa^{2} \tilde{\mathrm{H}}_{\tilde{\mathrm{G}}}
\end{aligned}
$$

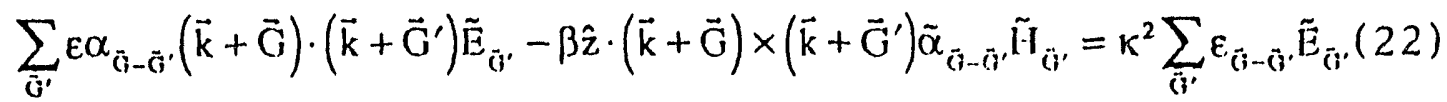

Eqs. (21) and (22) constitute a generalized eigenvalue equation of the form

$$
M\left(\begin{array}{l}
\tilde{E} \\
\tilde{H}
\end{array}\right)=\kappa^{2} \underset{N}{N}\left(\begin{array}{c}
\tilde{E} \\
\tilde{H}
\end{array}\right)
$$

Equation (23) can be brought to a symmetric, standard eigenvalue equation by making the substitution

$$
\tilde{\mathrm{E}}_{\tilde{\mathrm{G}}}=\left[\frac{1}{\sqrt{\varepsilon}}\right]_{\overline{\mathrm{G}}-\overline{\mathrm{G}}^{\prime}} \tilde{\mathrm{E}}_{\overline{\mathrm{G}}^{\prime}}^{\prime}
$$

For the case when $\beta=0$, Eqs. (21) and (22) are uncoupled, and using Eq. (24) reduce to the matrix equations for the unform polarizations reported previously.

The results of calculations for a square lattice are shown in Fig. 2(a)-(d), where we include the bandstructures for a selection of different $\beta$ values. For the $\beta=0$ case we plot the TM and TE modes separately. We note that for this particular example the $\beta=0$ TM mode band structure exhibits band gaps for all directions of $k$ vector, but that the $\beta=0$ TE mode band structure does not. Since the TE and TM modes hybridize for non-zero $\beta$, it is perhaps not surprising that none of the hybrid cases investigated show gaps. Even when both TE and TM bandstructures show gaps, the frequency range of the gaps may not overlap. Should this be the case it is likely that the gaps will be absent for non-zero $\beta$ cases. 


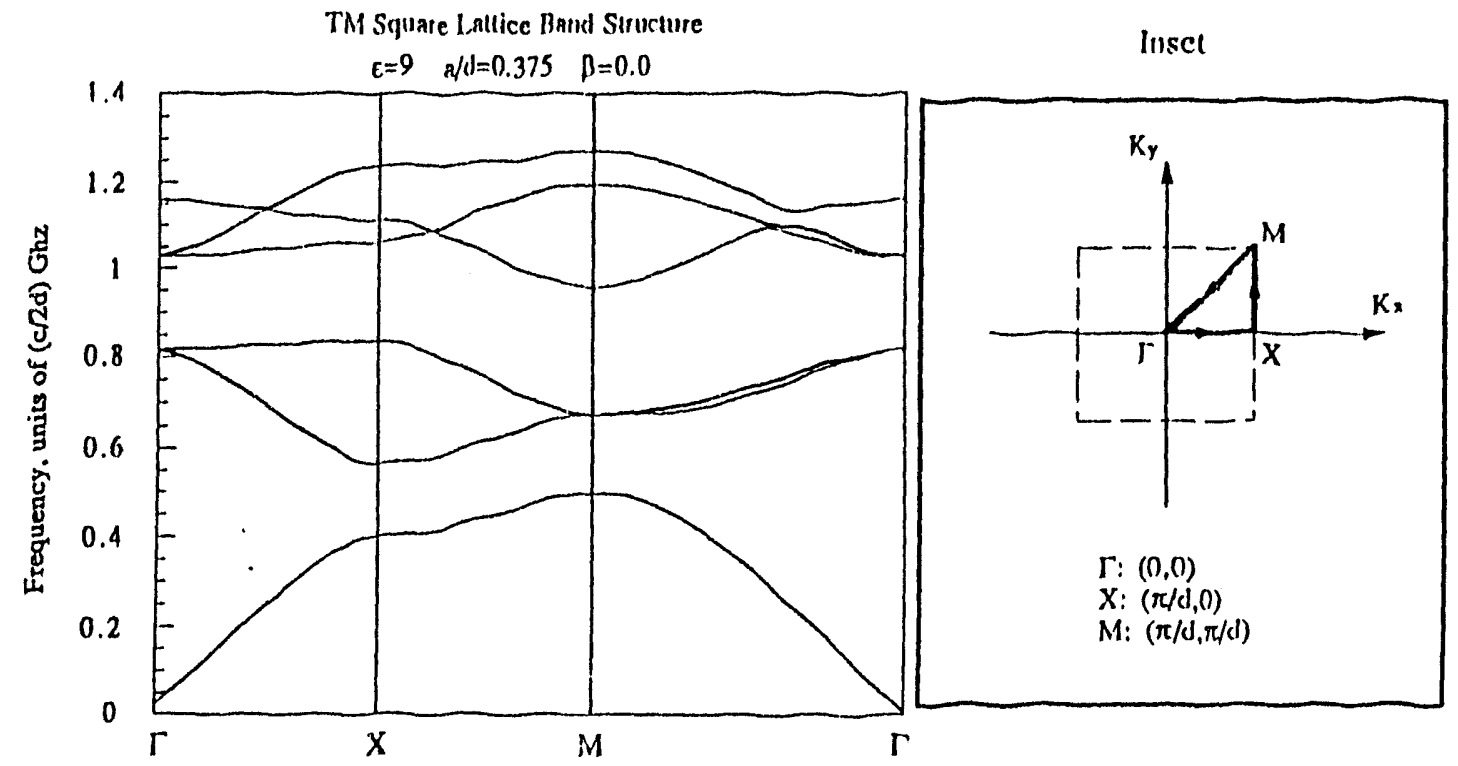

Fig. 2. (Inset) Because of the periodicity of the lattice, we may restrict our attention to frequencies corresponding to a mininum set of $k$ vectors. For a square lattice this sct, shown in the inset within the dashed lines, comprises also a square in reciprocal lattice space. Furthermore, because the real lattice has fourfold rotational and reflection symmetries, only the solutions for a single octumt of this square are unique. A plot of the frequencies corresponding to $k$ vectors along the boundary of the octant forms the "band structure". It is expected that each frequency span coincides with that of the full $\omega_{n}(\mathrm{k})$ surface. (a) Band structure for the square photonic lattice. In this case, the electric field is parallel to the cylinder axes (TM). There are two band gaps for this polarization evident for the given frequency range. 109 reciprocal lattice vectors were used to obtain the band structure plot.

TLi Square i.sllite Band Siruclume

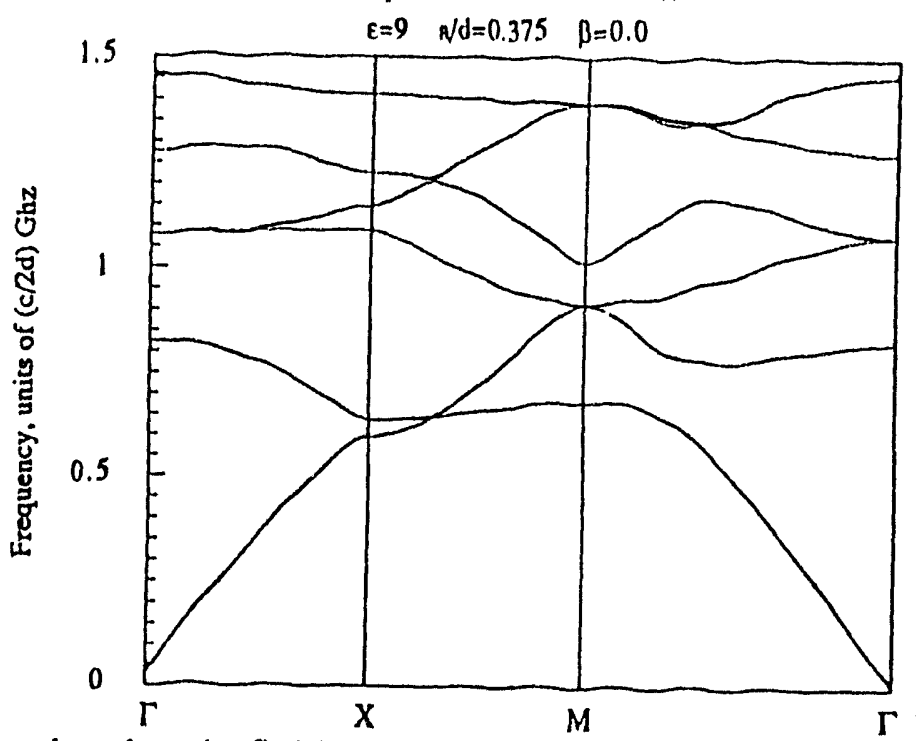

Fig. 2. (b) Here, the elcciric field is polarized perpendicular to the cylinder axes. There are no gaps in this case. 


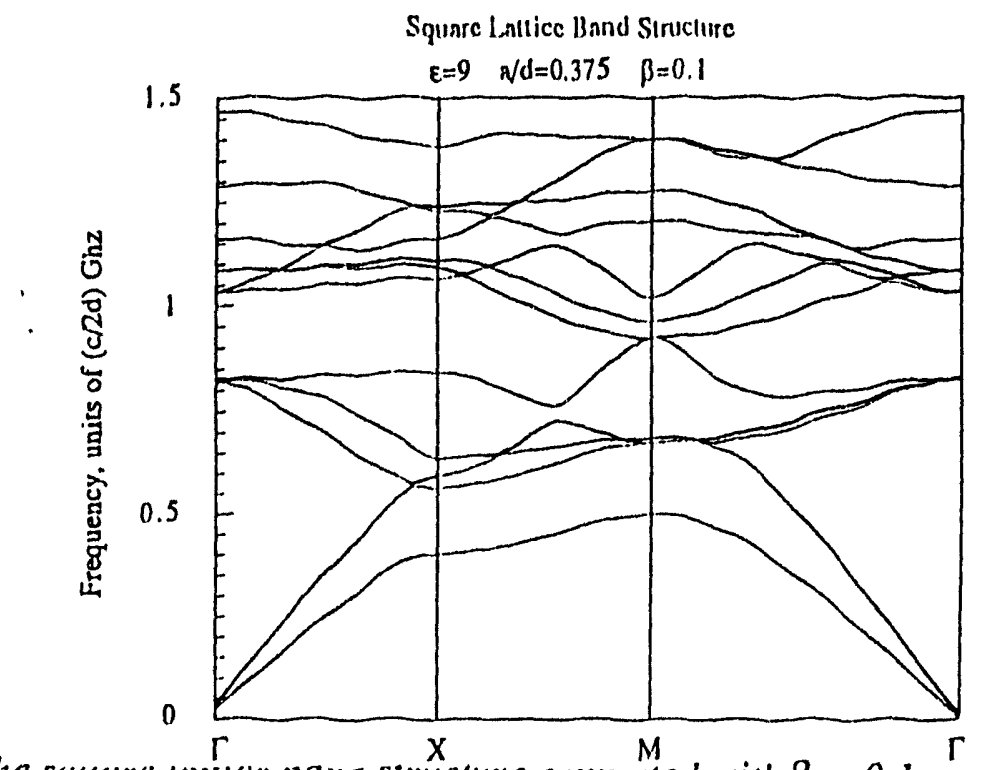

Fig.2 (c) The square catuce vana structure computed with $\beta=0.1$.

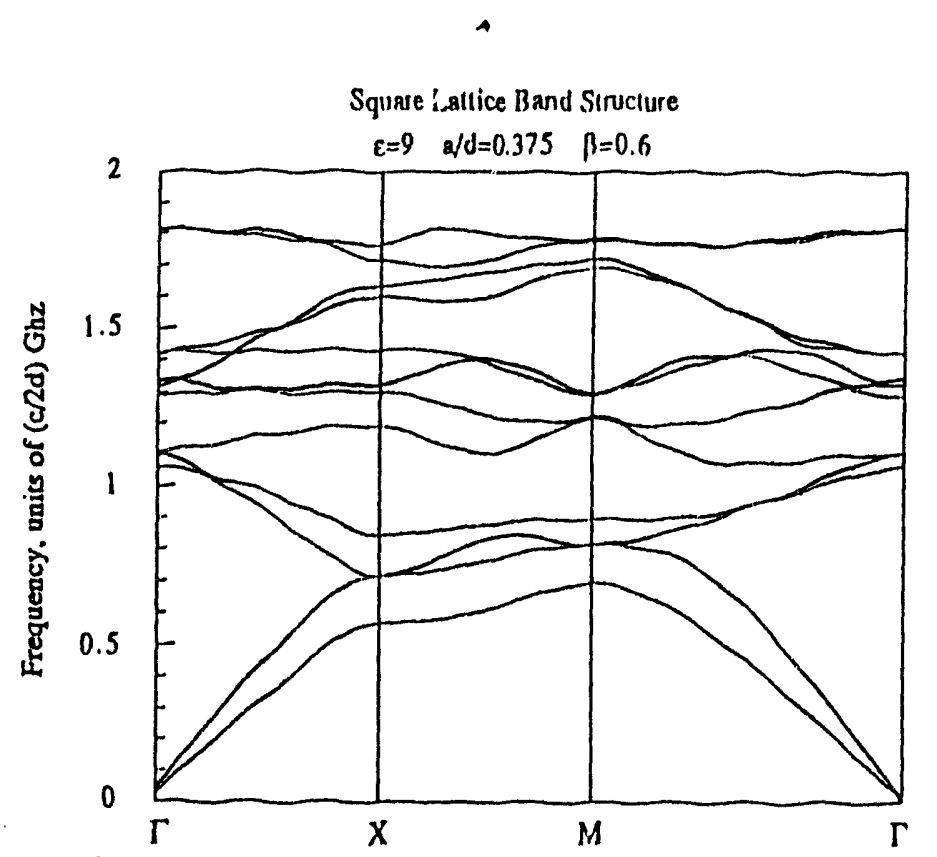

Fig. 2 (d) The square latticer band structure computed with $\beta=0.6$. 


\section{B. Defect Studies}

When the transhational symmetry of the lattice is violated by, for cxample, the addition of some impurity, previously forbidden states with imaginary values of the Bloch vector $k$ are now allowed. Thus, in addition to the exlended periodic rumning wave states which comprise the band structures for infinite latlices, there may also be states which decay away exponentially from the perturbed site. If the perturbation consists of a single cylinder removal, the associated mode is a defect mode. In 1-D it is possible to analyze defect states by directly assuming that they are formed from Bloch waves with imaginary values of $\mathrm{k}^{3}$

In higher dimensions the situation is more complicated, and we calculate the properties of impurity modes by expanding the new eigenfunctions in terms of the infinite lattice Bloch functions. Writing the diclectric function as

$$
\varepsilon(\vec{x})=\varepsilon_{\text {per }}(\vec{x})+\varepsilon_{\text {der }}(\vec{x})
$$

where we have separated the periodic variation from the local defect function, we may wrile Eq.(9) as

$$
\nabla^{2} E(\vec{x})+\frac{\omega^{2}}{c^{2}}\left(\varepsilon_{\text {ret }}(\vec{x})-1\right) E(\vec{x})+\frac{\omega^{2}}{c^{2}} E(\vec{x})=-\frac{\omega^{2}}{c^{2}} \varepsilon_{\text {iter }}(\vec{x}) \Gamma:(\vec{x})
$$

To arrive at Eq. (26) we set $\beta=0$, and concern ourselves only with the TM mode. We do this primarily because we do not have at hand a non-zero $\beta$ case for which a gap is present. Since the TM mode has E parallel to the cylinder axis, this is the mode of primary interest for accelerator applications. Because of the finite spacing between plates for the acceleritor cavity, only the $\Gamma M$ modes exist for $\beta=0$ ) for relevent frequencies.

Expanding the solution in terms of the Bloch waves found previously,

$$
\vec{E}(\vec{x})=\sum_{\vec{k}, n} f_{\vec{k}, n} E_{\ddot{k}, 11}(\vec{x})
$$

and using the orthogonality relation

$$
\int E_{\ddot{k}, n}^{*}(\vec{x}) \varepsilon_{p e r}(\vec{x}) E_{\vec{k}^{\prime}, n^{\prime}} d V=C_{\vec{k}_{, n}} \delta_{\vec{k} \vec{k}}, \delta_{n n^{\prime}}
$$

we find the eigenvalue equation

$$
\sum_{\vec{k}^{\prime}, n^{\prime}}\left[\int_{\substack{\text { defect } \\ \text { volume }}} d V E_{\vec{k}, n}^{*}(\vec{x}) \varepsilon_{\text {der }}(\vec{x}) E_{\vec{k}^{\prime}, n^{\prime}}\right] f_{\vec{k}^{\prime}, n^{\prime}}=\left[\frac{\omega_{\vec{k}, n}^{2}-\omega^{2}}{\omega^{2}}\right] f_{\vec{k}, n}
$$


where $n$ is the band index, and the normalization constant has been taken into the Bloch functions. Eq. (29) can be manipulated into a symmetric eigenvalue equation as

$$
\sum_{\vec{k}^{\prime}}\left[\frac{\delta_{\ddot{k}^{\prime}}}{\omega_{\vec{k}}^{2}}+\frac{M_{\ddot{k}^{\prime} \vec{k}^{\prime}}}{\omega_{\vec{k}^{\prime}} \omega_{\bar{k}^{\prime}}}\right] \alpha_{\vec{k}^{\prime}}=\frac{\alpha_{\ddot{k}^{\prime}}}{\omega^{2}}
$$

where

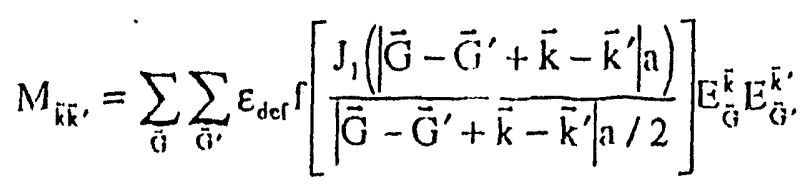

and

$$
\alpha_{\vec{k}}=\omega_{\vec{k}} f_{\vec{k}}
$$

We can use Eq. (30) to numerically calculate the modified band structure due to the addition of a perturbation in dielectric constant to an otherwise perfect lattice. In order to follow this procedure, however, we must first choose a finite set of $k$ values for the sum in Eq. (27). Chonsing a set of $\mathrm{N} \mathrm{k}$ values effectively constrains the size of the lattice to $\mathrm{N}$ sites; it is this sublattice, $\mathrm{N}$ sites plus the defect, that constitules the new unit cell of an overall super-latlice. We expect that for bound modes in which the fields fall off exponentially from the center of a perturbation, reasonable numerical convergence may be achieved with a fairly small number $N$ of siles.

To further reduce the computational task in the numerical calculation we can make use of any discrete rotational symmetry possessed by the impurity dielectric function. In the case of a defect in a squic lattice, for example, there are three distinct mode types; monopole, dipole, and quadrupole. An accelerator mode must, of course, have monopole character.

We have completed calculations for defects in square and triangular lattices utilizing the above procedure. We find, however, that the calculated defect frequency is very sensitive to the number of reciprocal lattice vectors (RLV's) used in the expansions in Eqs. (14) and (19). While the band structure calculation cxhibits reasonable convergence for the lower bands using only about one hundred RLV's, it appears that the equivalent convergence for the defect mode requires a much greater number of RLV's. Using an alternate technique, Meade et al. 8 have succeeded in calculating the frequency dependence of the defect modes in a square photonic lattice; their results agree very well with previously reported measurements .5

\section{EXPERIMENTAL RESULTS}

The focus of our experimental investigations has been to verify the various theoretical predictions resulting from the numerical calculations. Once the calculations demonstrate the necessary accuracy, it will then be possible to search for the optimal parameters for an accelerntor structure. In Fig. 3(a) we present transmission data for the triangular lattice, verifying the predicted second band gap. In Fig. 3(b) we present the transmission data for a triangular lattice with a defect (a 
single cylinder removed). Note the presence of the new peak in the forbidden region corresponding to the defect mode. The frequency width of the defect mode, zero in principle for an infinite lattice, in this case is set by the cylinder and boundary plate losses, in addition to the finite size of the lattice.

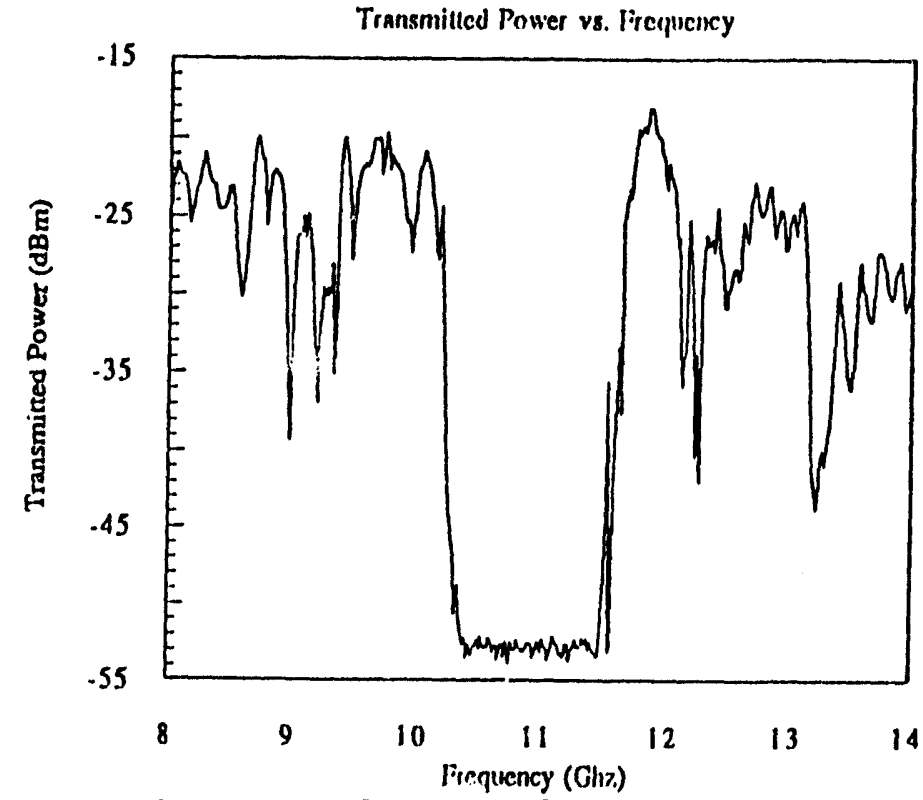

Fig. 3 (a): Transmitted power vs. frequnny for a triangular photonic lattice. The lattice was composed of 200 dielectric cylinders with $\varepsilon=9.0$ in a styrofoam template with $\varepsilon=1.03$. The lattice spacing was $1.27 \mathrm{~cm}$. In a transmission experiment, incident waves with frequencies corresponding to the forbidden band gap frequencies are exponentially attenuated across the lattice; thus, the sharp dip in transmitted power provides a measurement of a band gap from $10-11.5 \mathrm{GHz}$.

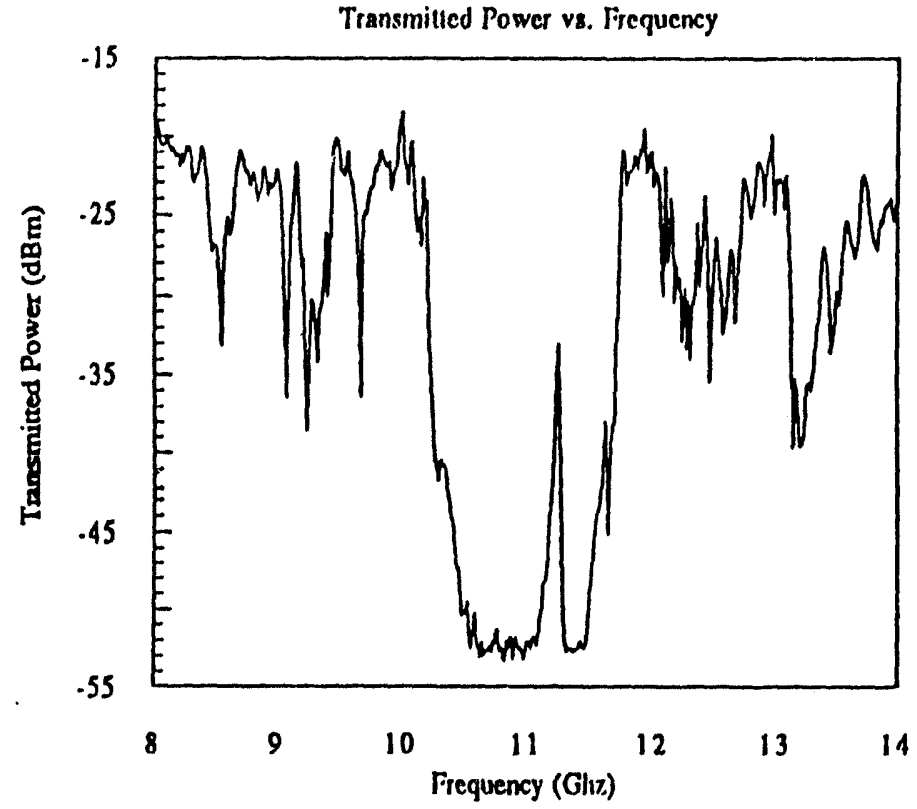

Fig. 3 (b) Transmitted power vs. frequency for a triangular lattice with a single cylinder removed. Note the new peak occurring in the band gap region corresponding to a defect mode. 
In Fig. 4 we show spatial mappings of the defect modes for square and triangular lattices with the same dielectric and lattice constants. We see that the triangular mode falls off much more rapidly away from the defect center than does the square lattice mode, a feature suggested by our computer simulations. The de: 1 modes in both cases are monopole in character.

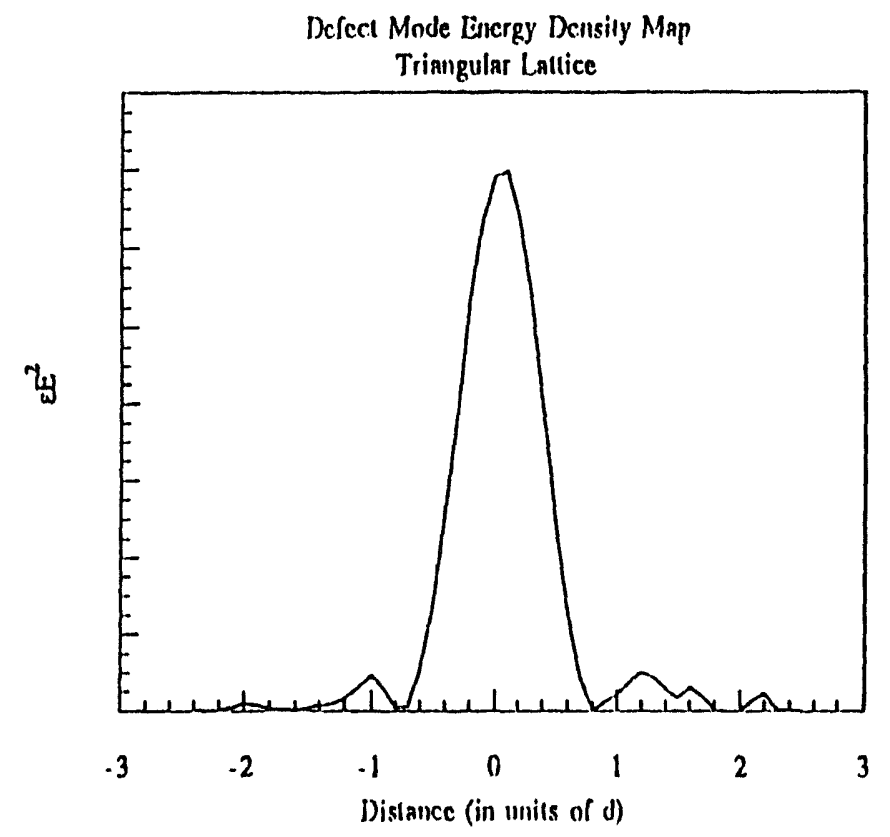

Fig. 4 (a) A map of the electric energy density for a defect mote in the second gap of the triangular lattice $(d=1.27 \mathrm{~cm})$. 


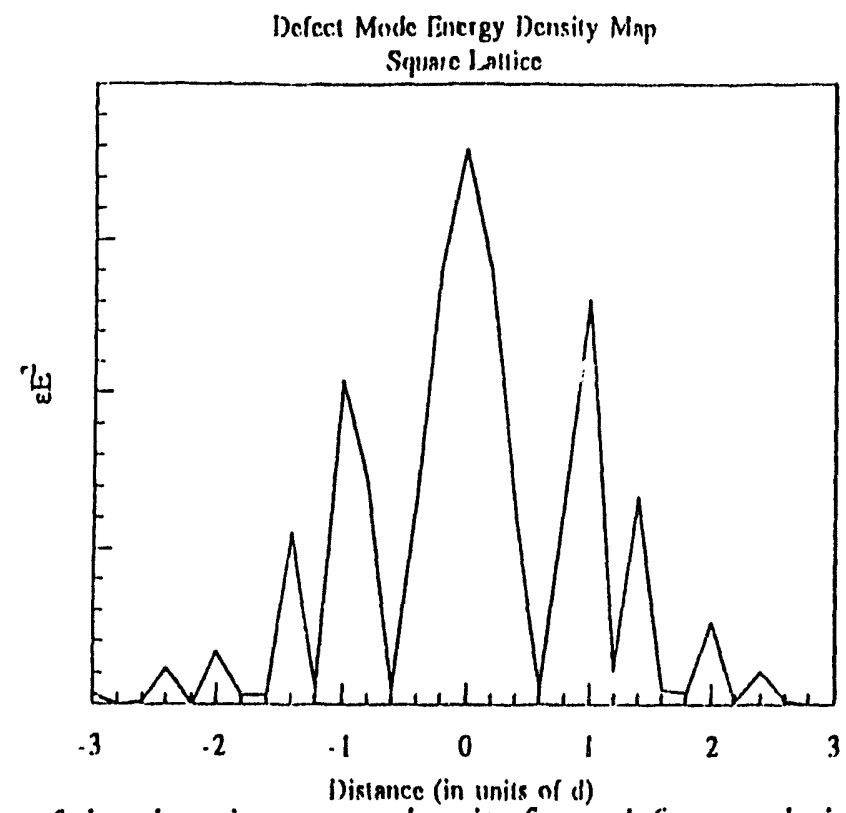

Fig. 4 (b) A map of the electric cnergy demsity for a defect mode in the second gap) of the square lattice $(d=1.50 \mathrm{~cm})$.

\section{CONCIJUDING DISCUSSION AND REMARKS}

In order to establish lic utility of PBG structures as accelerator cavities, a great deal more work needs to be done. We discuss in lhis section a number of issues (in addition to those mentioned in the introduction) with which we are currently concerned.

While we have shown the existence of trapped modes whose configuration appears to be suitable for particle acceleration, we need to investigate the question of higher order modes. It appears from the work allendy done that the exisicnce of band gaps is cxceptional rather than typical. We are hopeful, therefore, that structures can be found which exhibit a single band gap. Because the frequency range of the gap is limited, it seems likely that the number of trapped modes present in any gap is small, and our results scem to imply that situations can be found in which the gap contains only a single such mode. Given that this is the case, there remains the possibility that there will also exist quasi localized modes in the allowed frequency ranges. Such modes would be the analogue of the damped resonances of waveguide loatled conventional cavities. To sce how such modes might arise, recall that we found no band gaps for the finite q case for the square lattice. It is, however, very likely that a TM band gap mode is only slightly shifted and weakly coupled to propagating TE modes by a finite but small value of $\mathrm{q}$, leading 10 a mode of finite but high $\mathrm{Q}_{\mathrm{ex}}$. Such modes may also arise under circumstances in which the overlap range of two gaps is small. In this case propagation may occur only over a very limited range of angles in wave vector space. Localized modes with finite $Q_{e \times 1}$ may well occur in such regions, the narrow angle directions of propagation acting like a set of waveguides loading the cavity

In the text above we have only considered the use of metallic end walls as terminations of the PBG cavity in the $z$ direction. Mueller, et al. ${ }^{9}$ have suggested using the Clogston 10 layered conductor scheme as a means of reducing end wall losses without resorting to superconductivity. We also plan to investigate the utility of the Zakowicz ${ }^{11}$ dielectric coating scheme. We also need to investigate cavity-tocavity coupling. 
Apropos of the cavity coupling question, we are also considering the design of a band gap structure which avoids this issue. A smooth dielectric waveguide wilh $q c / \omega=1$ would be a suitable structure for an inverse Cerencov accelerator. On the basis of the results reported above for non-zero q, it seems unlikely that a low loss structure could be formed from a lattice array of parallel cylindrical rots. On the other hand it seems clear that a low loss radial band gap structure, of the sort mentionc i in the secriml section, can bc designed.

\section{ACKNOWLEDGEMENTS}

We thank Dr. F. M. Mueller for informative conversations. This research has been supported by the National Science Foundation, grant DMR-89-15815, and the Department of Energy, contracts DE-AS0389ER40527 and DE-AC03-76SF00515.

\section{REFERENCES}

1. S. John, Phys. Rev. Ictt. , 58, 2486 (1987).

2. E. Yablonovitch, Phy:. Rev. Lett., 58, 2059 (1987).

3. D. Smith, R. Dalichnouch, N. Kroll, S. Schultz, S. L. McCall, P. M. Platzman, "Photoric Band Structure and Defects in One-and Two-Dimensions", submitted to J. Opt. Soc. Amer.B (1992).

4. See also W. C. Sailor, F. M. Mueller, and B. E. Carlsten, "Theory for a cylindrical pillbox accelerator cavity using layered structures for reducing skin-effect losses", preprint (submitted to IEEE TMTT); and the presentation of Dr. R. B. Palmer at the 3rd Workshop on Advanced Accelerator Concepts, Port Jeficrson, NY June 14-20, 1992, entilled: "The Definition and Properties of Near Fields".

5. S. L. McCall, P. M. Platzman, R. Dalichaouch, D. Smith, S. Scluultz, Phy's. Rev. Lett., 67, 2017 (1991).

6. M. Plihal, A. Shambrook, A. A. Maradudin, Ping Sheng, Optics Communications, 80, 199 (1991).

7. M. Plihal, A. A. Maradudin, Phys. Rev. B, 44, 8565 (1991).

8. R. D. Meade, A. M. Rappe, K. D. Brommer, J. D. Joannopoulos, "Accurate Theoretical Analysis of Photonic Band-Gap Materials", preprint, submitted to Phys. Rev. Lett. (1992).

9. F. M. Mueller, private communication.

10. A. M. Clogton, Proc. of the I.R.E., pp. 767-782 (1951).

11. J. Zakowicz, J. Appl. Phys., 55, 3421 (1984). 

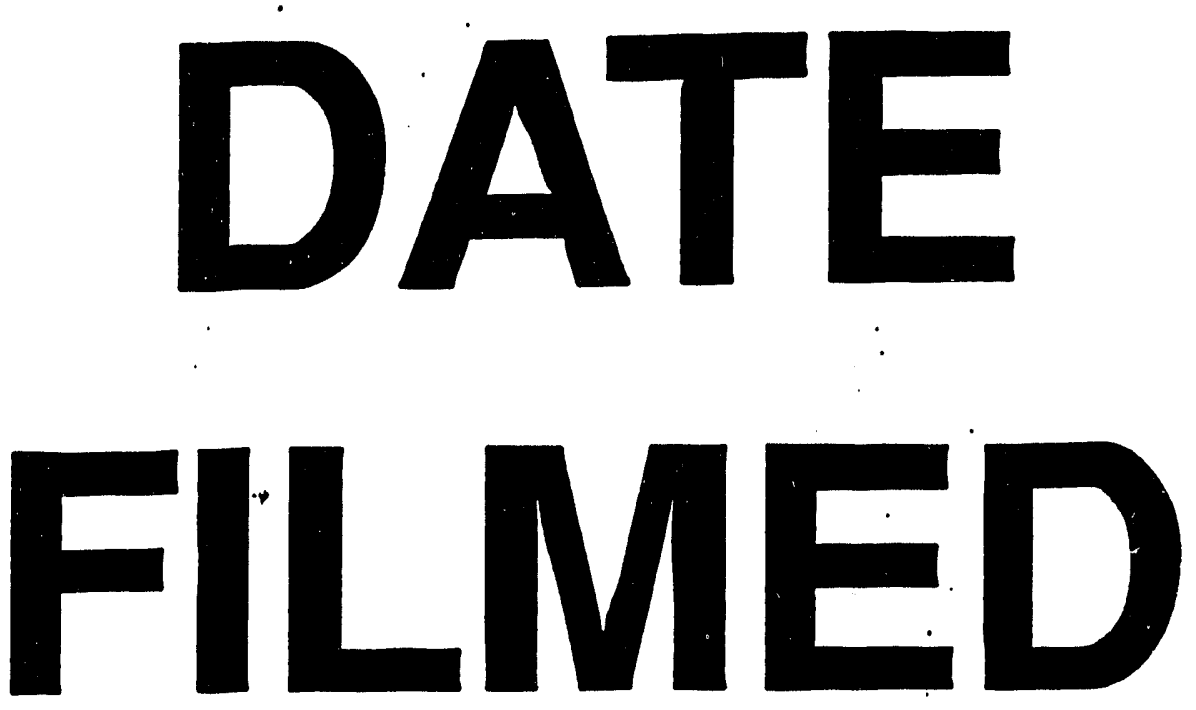

$12 / 29 / 93$
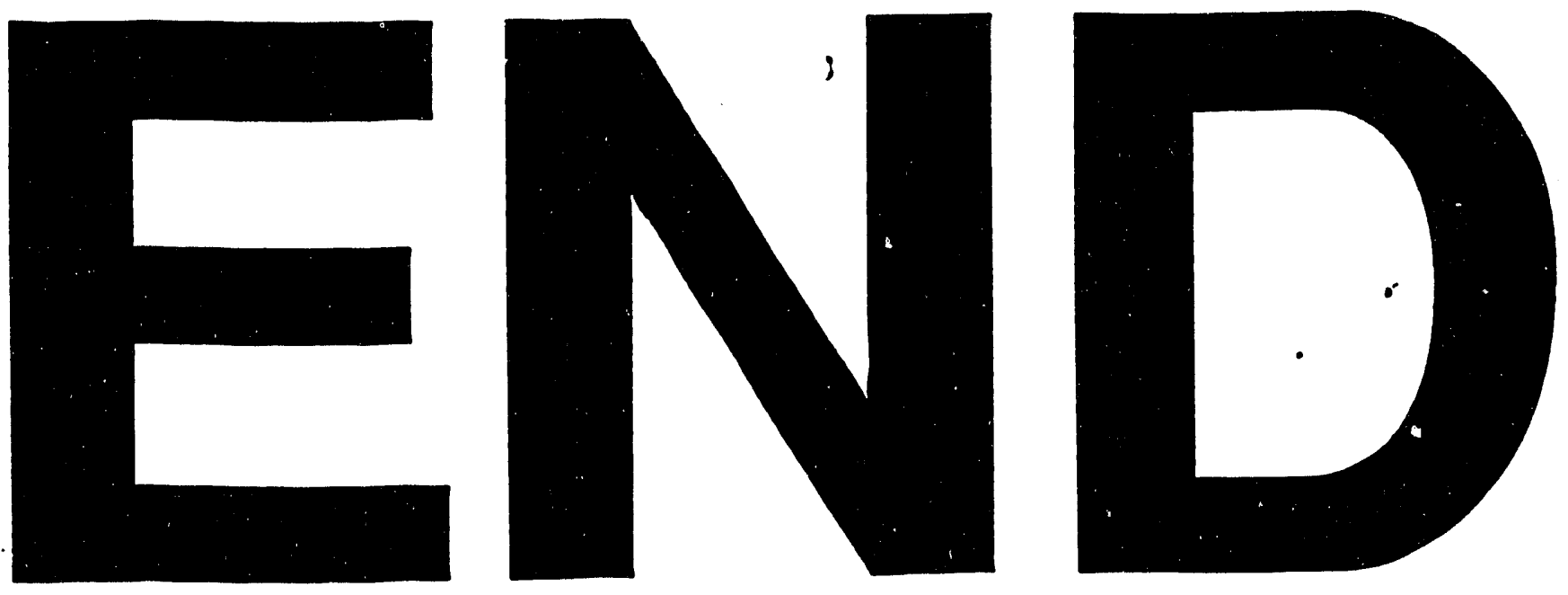
